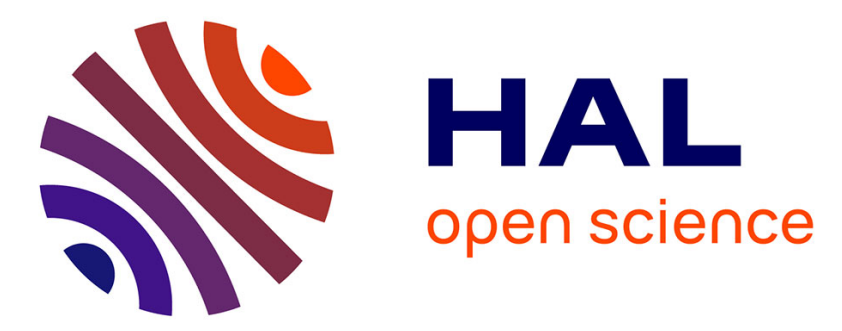

\title{
Fault-tolerant control of singularly perturbed systems with actuator faults and disturbances
}

Wu Yang, Yan-Wu Wang, Irinel-Constantin Morarescu, Zhi-Wei Liu, Yuehua Huang

\section{- To cite this version:}

Wu Yang, Yan-Wu Wang, Irinel-Constantin Morarescu, Zhi-Wei Liu, Yuehua Huang. Fault-tolerant control of singularly perturbed systems with actuator faults and disturbances. International Journal of Robust and Nonlinear Control, 2020, 30 (12), pp.4550-4564. 10.1002/rnc.4990 . hal-02545424

\section{HAL Id: hal-02545424 \\ https://hal.science/hal-02545424}

Submitted on 17 Apr 2020

HAL is a multi-disciplinary open access archive for the deposit and dissemination of scientific research documents, whether they are published or not. The documents may come from teaching and research institutions in France or abroad, or from public or private research centers.
L'archive ouverte pluridisciplinaire HAL, est destinée au dépôt et à la diffusion de documents scientifiques de niveau recherche, publiés ou non, émanant des établissements d'enseignement et de recherche français ou étrangers, des laboratoires publics ou privés. 


\title{
Fault-Tolerant Control of Singularly Perturbed Systems With Actuator Faults and Disturbances
}

\author{
Wu Yang ${ }^{123}$, Yan-Wu Wang ${ }^{12 *}$, Irinel-Constantin Morărescu ${ }^{4}$, Zhi-Wei Liu ${ }^{12}$, Yuehua \\ Huang $^{5}$ \\ ${ }^{1}$ School of Artificial Intelligence and Automation, Huazhong University of Science and Technology, Wuhan, 430074, \\ China \\ ${ }^{2}$ Key Laboratory of Image Processing and Intelligent Control (Huazhong University of Science and Technology), \\ Ministry of Education, Wuhan, 430074, China \\ ${ }^{3}$ School of Electrical and Electronic Engineering, Huazhong University of Science and Technology, Wuhan 430074, \\ China \\ ${ }^{4}$ Université de Lorraine, CNRS, CRAN, Nancy, 54000, France \\ ${ }^{5}$ College of Electrical Engineering and New Energy, China Three Gorges University, Yichang, 443002, China
}

\section{SUMMARY}

The paper proposes several fault-tolerant control (FTC) laws for singularly perturbed systems (SPS) with actuator faults and disturbances. On of the main challenges in this context is that the fast-slow decomposition is not available for actuator faults and disturbances. In view of this, some conditions for the asymptotic stability of the closed-loop dynamics are investigated by amending the composite Lyapunov approach. On top of this, a closed-form expression of the upper-bound of singular perturbation parameter (SPP) is provided. Moreover, we design several SPP-independent composite FTC laws which can be applied when this parameter is unknown. Finally, the chattering phenomenon is eliminated by using the continuous approximation technique. We also emphasize that, for linear SPSs, the FTC design can be formulated as a set of linear matrix inequalities while the SPP upper-bound can be obtained by solving a convex optimization problem. Two numerical examples are given to illustrate the effectiveness of the proposed methodology. Copyright (C) 0000 John Wiley \& Sons, Ltd.

Received ...

KEY WORDS: Singularly perturbed system; fault-tolerant control; actuator bias fault; disturbance.

\section{INTRODUCTION}

In response to ever-increasing need for stability and safety of the control systems, fault tolerant control (FTC) received a lot of attention over the past decades. There have been abundant and outstanding achievements on the FTC of various dynamical systems, such as uncertain linear/nonlinear systems $[1,2,3,4,5,6]$, switched systems $[7,8,9,10]$, fuzzy systems $[11,12,13]$, Markovian jump systems [14, 15, 16], singular systems [17, 18], multi-agent systems [19, 20], and so on.

While existing FTC techniques are developed for systems that evolve on a single time scale, real systems may involve processes that vary on multiple time-scales. Such systems can be found in various application areas such as power systems [21], mechanical systems [22], chemical reaction process [23], etc. In those cases the state variables can be divided into two types: slow and fast. To mathematically describe this complex dynamics one uses the framework of singularly perturbed systems (SPSs) which are analyzed by decoupling the slow and fast dynamics (see [24] for details).

*Correspondence to: Yan-Wu Wang (Email:wangyw@ hust.edu.cn) 
It is noteworthy that, the analysis and control synthesis design proposed for dynamical systems evolving on one time-scale are not directly applicable to SPSs. Indeed the presence of two timescales and of the singular perturbation parameter (SPP) characterizing the time scale separation will lead to numerical ill-conditioning and stiffness problems [24]. Consequently, the design of suitable FTC scheme for SPSs is an important and challenging problem that is addressed in this paper.

It is worth noting that several results on the FTC design for SPSs already exist in the literature. In [25] it is addressed the problem of FTC design for delayed SPSs with sensor fault while in [26], the authors considered the FTC design for nonlinear SPSs under actuator faults. As in many practical applications one has to also take into account external disturbances, [27] addressed the FTC design for linear SPSs face both actuator faults and external disturbances. Recently, Wang et al. [28] constructed a reliable FTC controller for discrete-time Takagi-Sugeno fuzzy SPSs with Markov jump topology. One of the main challenges in the FTC design for SPSs is the fact that the fast-slow decomposition for dynamics with actuator faults and disturbances is not available. While the control design proposed in [27, 28] depends on the SPP, this value cannot be accurately known in many applications. This is the reason why, we are proposing some SPP-independent FTC laws that guarantee asymptotic stability of the closed-loop even when the SPP is roughly known.

In this context we propose a FTC strategy for nonlinear SPSs that can deal with both actuator faults and external disturbances. By employing composite Lyapunov function method, we propose conditions guaranteeing asymptotic stability of the closed-loop dynamics. Moreover we derive the upper-bound of SPP for which the aforementioned conditions hold. On top of that, several SPP-independent composite FTC laws are proposed. To avoid the chattering phenomenon, the designed FTC laws are further improved by using continuous approximation method. To sum up, the contributions of this work are summarized as follows:

1. We propose a general FTC design framework for a class of nonlinear SPS in presence of actuator faults and external disturbances, which cannot be decomposed according to the fastslow dynamics.

2. The proposed composite FTC laws consist of a composite controller designed to ensure the stability of the nominal model, and a compensation law used to suppress the effects of the external disturbance and actuator fault. Moreover, several SPP-independent composite FTC laws are developed to reduce the design complexity and expand their applications.

3. Unlike [25, 26, 27], we further provide an upper-bound on SPP guaranteeing that our results can be applied.

Notations: $\mathbb{R}^{n}$ is the set of real $n$ dimensional vectors. A real symmetric matrix $P$ which is negative (positive) definite is denoted by $P<(>) 0$. $\operatorname{He}\{A\}$ denotes $A+A^{T}$. The symbol $*$ denotes the symmetric element in block matrix. A function $\phi:[0,+\infty) \mapsto[0,+\infty)$ belongs to class $K_{\infty}$ if $\phi$ is continuous, strictly increasing and $\phi(0)=0$.

\section{FTC FOR NONLINEAR SPS}

This section provides results concerning the FTC design for a general class of nonlinear SPSs with actuator faults and disturbances. Furthermore, we provide a closed form characterization of an upper-bound on the SPP that guarantee the proposed FTC design is effective.

\subsection{Nonlinear SPS With Actuator Fault and Disturbances}

Consider the following class of nonlinear SPSs with the external disturbance and the actuator fault:

$$
\left\{\begin{array}{l}
\dot{x}=f_{1}(x, z)+g_{1}(x, z)\left(u+F_{a}\right)+\varrho_{1}(x, z) w \\
\varepsilon \dot{z}=f_{2}(x, z)+g_{2}(x, z)\left(u+F_{a}\right)+\varrho_{2}(x, z) w
\end{array}\right.
$$

where $x \in B_{x} \subset \mathbb{R}^{n_{x}}$ is the slow state, $z \in B_{z} \subset \mathbb{R}^{n_{z}}$ is the fast state, $u \in B_{u} \subset \mathbb{R}^{n_{u}}$ is the control input, and $w \in \mathbb{R}^{n_{w}}$ represents the external disturbance with the upper bound $\bar{w}$, i.e., $\|w\| \leq \bar{w}$. SPP $\varepsilon>0$ characterizes the difference of speed variation between the fast and slow dynamics. The 
nonlinear functions $f_{i}(\cdot, \cdot), g_{i}(\cdot, \cdot) \neq 0$, and $\varrho_{i}(\cdot, \cdot)$ are locally Lipschitz in $B_{x} \times B_{z} . F_{a}$ represents the actuator bias fault.

Remark 1: Note that the FTC design problem for nonlinear SPSs was also investigated in [26]. Unlike [26] we consider a more general class of dynamics that presents both external disturbances and actuator faults. In particular the external disturbances $\varrho_{1}(x, z) w$ and $\varrho_{2}(x, z) w$ are not limited to be matched. Moreover, we go beyond the results proposed in [26] by proposing an explicit upperbound on the SPP that guaranties the FTC design will be efficient.

Throughout this paper, the following assumption is made.

Assumption 1 The actuator bias fault is bounded, i.e., there exists positive constant $\bar{F}$ such that $\left\|F_{a}\right\| \leq \bar{F}$.

Remark 2: Assumption 1 is quite natural and is common in the FTC literature, such as [26, 29]. The main objective of this work is to solve the following problem.

Control Problem: Design an SPP-independent FTC law for singularly perturbed nonlinear system (1) that guaranty the asymptotic stability of the closed-loop in presence of actuator faults and external disturbances.

\subsection{Composite FTC Schemes}

In this subsection, the composite FTC law is constructed as:

$$
u=u_{n c}+u_{a} .
$$

Remark 3: The proposed FTC law $u$ consists of two parts, a composite control law $u_{n c}$ and the compensate control law $u_{a}$. The former is developed based on the nominal model and is designed to achieve the desirable stability of the nominal model, while the latter is used to eliminate the effect of the actuator fault and the external disturbances.

The nominal open-loop system associated with system (1) is defined as

$$
\left\{\begin{array}{l}
\dot{x}=f_{1}(x, z)+g_{1}(x, z) u_{n c} \\
\varepsilon \dot{z}=f_{2}(x, z)+g_{2}(x, z) u_{n c}
\end{array}\right.
$$

where $u_{n c}$ is the composite nominal control law for system (3). Assume that system (3) is a standard SPS for each $u_{n c} \in B_{u} \subset \mathbb{R}^{n_{u}}$, that is, the equation $0=f_{2}(x, z)+g_{2}(x, z) u_{n c}$ owns a unique solution $z=h\left(x, u_{n c}\right)$ in $B_{x} \times B_{z} \times B_{u}$.

Let $u_{n c}=u_{s}+u_{f}$, where $u_{s}$ is the control law depends on the slow state variable $x$, while $u_{f}$ is the control law depends on both the state variables $x$ and $z$. Specifically, $u_{s}$ and $u_{f}$ can be expressed as $u_{s}=K_{s}(x)$ and $u_{f}=K_{f}(x, z)$, respectively. As pointed out in [30], $u_{f}=K_{f}(x, z)$ is such that 1) $z=H(x) \triangleq h\left(x, K_{s}(x)\right)$ defined on $B_{x} \times B_{z}$ is a a unique solution of $f_{2}(x, z)+$ $\left.g_{2}(x, z)\left(K_{s}(x)+K_{f}(x, z)\right)=0 ; 2\right) K_{f}(x, H(x))=0$.

Following standard developments in the analysis of SPSs, the reduced order subsystem is described by:

$$
\dot{x}=f_{1}\left(x, h\left(x, u_{s}\right)\right)+g_{1}\left(x, h\left(x, u_{s}\right)\right) u_{s},
$$

and the boundary layer subsystem by:

$$
\frac{d z}{d \tau}=f_{2}(x, z)+g_{2}(x, z)\left(u_{s}+u_{f}\right)
$$

It is noteworthy that $x$ is treated as a fixed parameter in the boundary layer subsystem (5), the expanded time variable can be represented as $\tau=t / \varepsilon$.

The design procedure of the composite FTC law (2) can be summarized as follows.

Step 1: Design the slow control law $u_{s}=K_{s}(x)$ such that the controlled reduced order subsystem (4) is asymptotically stable. Then, there exist a Lyapunov function $V(x)$ and a positive constant $\alpha_{1}$ such that for all $x \in B_{x}$

$$
\frac{\partial V}{\partial x}\left[f_{1}(x, H(x))+g_{1}(x, H(x)) K_{s}(x)\right] \leq-\alpha_{1} \zeta^{2}(x),
$$


where $\zeta(x) \in K_{\infty}$.

Step 2: With the selected slow control law $u_{s}=K_{s}(x)$, design the fast control law $u_{f}=K_{f}(x, z)$ such that the boundary layer subsystem (5) has a globally asymptotically stable equilibrium $z=H(x)$, which implies that there exist a Lyapunov function $W(x, z)$ and a positive constant $\alpha_{2}$ such that for all $(x, z) \in B_{x} \times B_{z}$

$$
\frac{\partial W}{\partial z}\left[f_{2}(x, z)+g_{2}(x, z)\left(K_{s}(x)+K_{f}(x, z)\right)\right] \leq-\alpha_{2} \eta^{2}(z, H)
$$

where $\eta(z, H)=\eta(z-H(x)) \in K_{\infty}$.

Step 3: Choose proper constants $\beta_{1}, \beta_{2}$ and $\gamma$ to ensure the following interconnection conditions hold

$$
\begin{aligned}
& \frac{\partial V}{\partial x}\left[f_{1}(x, z)+g_{1}(x, z)\left(K_{s}(x)+K_{f}(x, z)\right)\right. \\
& \left.\quad-f_{1}(x, H(x))-g_{1}(x, H(x)) K_{s}(x)\right] \leq \beta_{1} \zeta(x) \eta(z, H) \\
& \frac{\partial W}{\partial x}\left[f_{1}(x, z)+g_{1}(x, z)\left(K_{s}(x)+K_{f}(x, z)\right)\right] \leq \gamma \eta^{2}(z, H)+\beta_{2} \zeta(x) \eta(z, H) .
\end{aligned}
$$

Step 4: Design the compensate control law $u_{a}$

$$
u_{a}=-\frac{\Pi^{T} \bar{F}}{\|\Pi\|}-\frac{\Pi^{T}\|\Psi\| \bar{w}}{\|\Pi\|^{2}}
$$

where $\Pi=(1-\theta) R_{1} g_{1}(x, z)+\theta R_{2} g_{2}(x, z)+\theta \varepsilon R_{3} g_{1}(x, z), \theta \in(0,1), \Psi=(1-\theta) R_{1} \varrho_{1}(x, z)+$ $\theta R_{2} \varrho_{2}(x, z)+\theta \varepsilon R_{3} \varrho_{1}(x, z), R_{1} \triangleq \frac{\partial V}{\partial x}, R_{2} \triangleq \frac{\partial W}{\partial z}, R_{3} \triangleq \frac{\partial W}{\partial x}$.

\subsection{Stability Analysis}

Theorem 1: Consider nonlinear SPS (1) with Assumption 1 is fulfilled. If the composite FTC law (2) is designed based on Steps $1-4$, system (1) is asymptotically stable for any $\varepsilon \in\left(0, \varepsilon^{*}\right)$, where $\varepsilon^{*}$ can determined as follows:

- If $\gamma=0$, then

$$
\varepsilon^{*}=\left\{\begin{array}{l}
+\infty, \text { if } \beta_{2}=0, \chi\left(\theta, \alpha_{1}, \alpha_{2}, \beta_{1}\right)>0 \\
\frac{-(1-\theta) \beta_{1}+2 \sqrt{\theta(1-\theta) \alpha_{1} \alpha_{2}}}{\theta \beta_{2}}, \text { if } \beta_{2} \neq 0
\end{array}\right.
$$

- If $\gamma \neq 0$, then

$$
\varepsilon^{*}=\left\{\begin{array}{l}
\frac{\chi\left(\theta, \alpha_{1}, \alpha_{2}, \beta_{1}\right)}{\theta \alpha_{1} \gamma}, \text { if } \beta_{2}=0, \chi\left(\theta, \alpha_{1}, \alpha_{2}, \beta_{1}\right)>0 \\
\frac{-b+\sqrt{b^{2}-4 a c}}{2 a}, \text { if } \beta_{2} \neq 0
\end{array}\right.
$$

with $\quad \chi\left(\theta, \alpha_{1}, \alpha_{2}, \beta_{1}\right)=\theta \alpha_{1} \alpha_{2}-0.25(1-\theta) \beta_{1}^{2}, \theta \in(0,1), a=\theta^{2} \beta_{2}^{2}, b=2 \theta(1-\theta)\left(\alpha_{1} \gamma+\right.$ $\left.2 \beta_{1} \beta_{2}\right), c=(1-\theta)^{2} \beta_{1}^{2}-4 \theta(1-\theta) \alpha_{1} \alpha_{2}$.

Proof: Define the composite Lyapunov function candidate $v(x, z)=(1-\theta) V(x)+\theta \varepsilon W(x, z)$, where $\theta \in(0,1)$ and Lyapunov functions $V(x)$ and $W(x, z)$ are as in Step 1 and Step 2. Then, the 
time derivative of $v(x, z)$ along the trajectories of system (1) is given by

$$
\begin{aligned}
\dot{v}(x, z)= & (1-\theta) \frac{\partial V}{\partial x}\left[f_{1}(x, H(x))+g_{1}(x, H(x)) K_{s}(x)\right] \\
& +\theta \frac{\partial W}{\partial z}\left[f_{2}(x, z)+g_{2}(x, z)\left(K_{s}(x)+K_{f}(x, z)\right)\right] \\
& +(1-\theta) \frac{\partial V}{\partial x}\left[f_{1}(x, z)+g_{1}(x, z)\left(K_{s}(x)+K_{f}(x, z)\right)\right. \\
& \left.-f_{1}(x, H(x))-g_{1}(x, H(x)) K_{s}(x)\right] \\
& +\theta \varepsilon \frac{\partial W}{\partial x}\left[f_{1}(x, z)+g_{1}(x, z)\left(K_{s}(x)+K_{f}(x, z)\right)\right] \\
& +(1-\theta) \frac{\partial V}{\partial x} g_{1}(x, z)\left(u_{a}+F_{a}\right)+\theta \frac{\partial W}{\partial z} g_{2}(x, z)\left(u_{a}+F_{a}\right) \\
& +\theta \varepsilon \frac{\partial W}{\partial x} g_{1}(x, z)\left(u_{a}+F_{a}\right)+(1-\theta) \frac{\partial V}{\partial x} \varrho_{1}(x, z) w \\
& +\theta \frac{\partial W}{\partial z} \varrho_{2}(x, z) w+\theta \varepsilon \frac{\partial W}{\partial x} \varrho_{1}(x, z) w .
\end{aligned}
$$

It follows from the conditions (6)-(9) that

$$
\begin{aligned}
\dot{v}(x, z) \leq & -(1-\theta) \alpha_{1} \zeta^{2}(x)-\theta \alpha_{2} \eta^{2}(z, H)+(1-\theta) \beta_{1} \zeta(x) \eta(z, H) \\
& +\theta \varepsilon \beta_{2} \zeta(x) \eta(z, H)+\theta \varepsilon \gamma \eta^{2}(z, H)+(1-\theta) \frac{\partial V}{\partial x} g_{1}(x, z)\left(u_{a}+F_{a}\right) \\
& +\theta \frac{\partial W}{\partial z} g_{2}(x, z)\left(u_{a}+F_{a}\right)+\theta \varepsilon \frac{\partial W}{\partial x} g_{1}(x, z)\left(u_{a}+F_{a}\right) \\
& +(1-\theta) \frac{\partial V}{\partial x} \varrho_{1}(x, z) w+\theta \frac{\partial W}{\partial z} \varrho_{2}(x, z) w+\theta \varepsilon \frac{\partial W}{\partial x} \varrho_{1}(x, z) w \\
\triangleq & {\left[\begin{array}{c}
\zeta \\
\eta
\end{array}\right]^{T} \Omega(\varepsilon)\left[\begin{array}{l}
\zeta \\
\eta
\end{array}\right]+\Pi\left(u_{a}+F_{a}\right)+\Psi w, }
\end{aligned}
$$

where $\Omega(\varepsilon)=\left[\begin{array}{cc}-(1-\theta) \alpha_{1} & \frac{(1-\theta) \beta_{1}+\theta \varepsilon \beta_{2}}{2} \\ * & \theta \varepsilon \gamma-\theta \alpha_{2}\end{array}\right]$.

Substituting the compensate control law (10) into the above inequality yields

$$
\dot{v}(x, z) \leq\left[\begin{array}{l}
\zeta \\
\eta
\end{array}\right]^{T} \Omega(\varepsilon)\left[\begin{array}{l}
\zeta \\
\eta
\end{array}\right]
$$

Note that for any $\varepsilon \in\left(0, \varepsilon^{*}\right)$, the matrix $\Omega(\varepsilon)$ is a Hurwitz matrix. Then, it can be concluded that for a sufficient small positive scalar $\delta$,

$$
\dot{v}(x, z) \leq-\delta\left(\|\zeta\|^{2}+\|\eta\|^{2}\right)<0, \forall \varepsilon \in\left(0, \varepsilon^{*}\right) .
$$

Which means the origin of system (1) is asymptotically stable for any $\varepsilon \in\left(0, \varepsilon^{*}\right)$. The proof is thus completed.

We notice that the compensate control law $u_{a}$ proposed in (10) relies on the accurate knowledge of the SPP. Consequently, the proposed FTC law cannot be applied in many applications in which the SPP cannot be obtained precisely. To overcome this drawback, when the SPP is unknown but sufficiently small, an SPP-independent FTC law is given as $\bar{u}_{c}=u_{n c}+\bar{u}_{a}$, where

$$
\bar{u}_{a}=-\frac{\bar{\Pi}^{T} \bar{F}}{\|\bar{\Pi}\|}-\frac{\bar{\Pi}^{T}\|\bar{\Psi}\| \bar{w}}{\|\bar{\Pi}\|^{2}},
$$

where $\bar{\Pi}=(1-\theta) R_{1} g_{1}(x, z)+\theta R_{2} g_{2}(x, z), \bar{\Psi}=(1-\theta) R_{1} \varrho_{1}(x, z)+\theta R_{2} \varrho_{2}(x, z)$.

As a result, the following corollary can be achieved directly. 
Corollary 1: Under Assumption 1, the FTC law $\bar{u}_{c}$ asymptotically stabilizes the nonlinear SPS (1) as far as $\varepsilon$ is unknown but sufficiently small.

Note that the composite FTC laws $u_{c}$ and $\bar{u}_{c}$ will lead to the undesirable chattering phenomenon due to the discontinuous of the compensate control law. To mitigate the fluctuation of the actuator, by utilizing the continuous approximation technique, the composite FTC laws $\bar{u}_{c}$ can be replaced by

$$
\hat{u}_{c}=\left\{\begin{array}{l}
u_{n c}+\bar{u}_{a}, \text { if }\|\bar{\Pi}\| \geq \mu, \\
u_{n c}-\frac{\bar{\Pi}^{T} \bar{F}}{\mu}-\frac{\bar{\Pi}^{T}\|\bar{\Psi}\| \bar{w}}{\mu^{2}}, \text { otherwise. }
\end{array}\right.
$$

where $\mu$ is a predefined small positive constant.

Applying the composite FTC law (14) to system (1), according to the classical analysis result [30], the solutions of the closed-loop system are only uniformly ultimately bounded, and their bounds depend on the parameter $\mu$.

\section{FTC FOR LINEAR SPS}

This section is motivated by the fact that for nonlinear systems the construction of an appropriate Lyapunov function remains a challenging problem. Here, we provide several efficient FTC schemes for a class of linear SPSs. The most attractive feature of the obtained result in this section is that FTC schemes are provided as a set of linear matrix inequalities (LMIs) which are numerically tractable and the upper bound of $\varepsilon$ can also be determined by solving an optimization problem.

\subsection{Linear SPSs With Actuator Fault and Disturbances}

Consider a class of linear SPSs in the presence of actuator bias faults and disturbances, which is given by

$$
\left\{\begin{array}{l}
\dot{x}=A_{11} x+A_{12} z+B_{1}\left(u+F_{a}\right)+D_{1} w \\
\varepsilon \dot{z}=A_{21} x+A_{22} z+B_{2}\left(u+F_{a}\right)+D_{2} w,
\end{array}\right.
$$

where $x \in \mathbb{R}^{n_{x}}, z \in \mathbb{R}^{n_{z}}, u \in \mathbb{R}^{n_{u}}$ and $w \in \mathbb{R}^{n_{w}}$ are the slow state, the fast state, the control input, and the external disturbance, respectively. $F_{a}$ is again the actuator bias fault and satisfies Assumption 1 . For any $i, j=1,2, A_{i j}, B_{i}$ and $D_{i}$ are known real constant matrices with appropriate dimensions. Here, we do not require that $D_{i}=B_{i} G, i=1,2$, where the matrix $G$ is of appropriate dimensions, which means that the disturbances considered in this paper are not limited to the matched external disturbances.

The nominal model of system (15) can be expressed as

$$
\left\{\begin{array}{l}
\dot{x}=A_{11} x+A_{12} z+B_{1} u_{n c} \\
\varepsilon \dot{z}=A_{21} x+A_{22} z+B_{2} u_{n c}
\end{array}\right.
$$

Assume that system (16) is a standard SPS, that is, $A_{22}$ is nonsingular. System (16) is assumed to be strongly controllable. Moreover, the reduced order subsystem of system (16) is given as follows

$$
\left\{\begin{array}{l}
\dot{x}_{s}=A_{0} x_{s}+B_{0} u_{s} \\
z_{s}=-A_{22}^{-1}\left(A_{21} x_{s}+B_{2} u_{s}\right)
\end{array}\right.
$$

where $x_{s}, z_{s}$ and $u_{s}$ are the slow components of the corresponding variables $x, z$ and $u, A_{0}=$ $A_{11}-A_{12} A_{22}^{-1} A_{21}, B_{0}=B_{1}-A_{12} A_{22}^{-1} B_{2}$, and the boundary layer subsystem of system (16) is given as follows

$$
\frac{d z_{f}}{d \tau}=A_{22} z_{f}+B_{2} u_{f}
$$

where $z_{f}=z-z_{s}$ and $u_{s}=u-u_{s}$ are the fast components of the corresponding variables $x$ and $u, \tau=t / \varepsilon$ is the fast time scale variable. 


\subsection{Composite FTC Schemes}

Since system (16) is strongly controllable, then, both $\left(A_{0}, B_{0}\right)$ and $\left(A_{22}, B_{2}\right)$ are controllable. Which implies that there exist the gain matrices $K_{0}, K_{2}$ and positive definite matrices $P_{0}, P_{2}$ such that $A_{0}+B_{0} K_{0}$ and $A_{22}+B_{2} K_{2}$ are Hurwitz and satisfy the following LMIs:

$$
\begin{aligned}
\operatorname{He}\left\{P_{0}\left(A_{0}+B_{0} K_{0}\right)\right\} & <0, \\
\operatorname{He}\left\{P_{2}\left(A_{22}+B_{2} K_{2}\right)\right\} & <0 .
\end{aligned}
$$

Therefore, the slow control law and the fast control law can be designed as $u_{s}=K_{0} x_{s}$ and $u_{f}=K_{2} z_{f}$, respectively. Then, combining $u_{s}$ and $u_{f}$, the composite control law $u_{n c}$ for the nominal model (16) is represented as

$$
u_{n c}=K_{1} x+K_{2} z,
$$

where $K_{1}=\left(I_{n}+K_{2} A_{22}^{-1} B_{2}\right) K_{0}+K_{2} A_{22}^{-1} A_{21}$.

Furthermore, the compensate control law $u_{a}$ is designed as

$$
u_{a}=-\frac{\tilde{\Pi}^{T} \bar{F}}{\|\tilde{\Pi}\|}-\frac{\tilde{\Pi}^{T}\|\tilde{\Psi}\| \bar{w}}{\|\tilde{\Pi}\|^{2}},
$$

where $\quad \tilde{\Pi}=2\left(\phi^{T} P_{0} \bar{B}_{1}+\psi^{T} P_{2} \bar{B}_{2}\right), \quad \tilde{\Psi}=2\left(\phi^{T} P_{0} \bar{D}_{1}+\psi^{T} P_{2} \bar{D}_{2}\right), \quad \bar{B}_{1}=B_{1}-\bar{H} B_{2}-$ $\varepsilon \bar{H} \bar{L} B_{1}, \bar{B}_{2}=B_{2}+\varepsilon \bar{L} B_{1}, \quad \bar{D}_{1}=D_{1}-\bar{H} D_{2}-\varepsilon \bar{H} \bar{L} D_{1}, \bar{D}_{2}=D_{2}+\varepsilon \bar{L} D_{1}, \quad \bar{H}=\bar{A}_{12} \bar{A}_{22}^{-1}, \bar{L}=$ $\bar{A}_{22}^{-1} \bar{A}_{21}, \bar{A}_{i j}=A_{i j}+B_{i} K_{j}, i, j=1,2, \phi$ and $\psi$ satisfy

$$
\left[\begin{array}{l}
\phi \\
\psi
\end{array}\right]=\left[\begin{array}{cc}
I-\varepsilon \bar{H} \bar{L} & -\varepsilon \bar{H} \\
\bar{L} & I
\end{array}\right]\left[\begin{array}{l}
x \\
z
\end{array}\right] .
$$

Next, the main result on the asymptotic stability of the closed-loop system (15) is given.

\subsection{Stability Analysis}

Theorem 2: Consider linear SPS (15) with Assumption 1 is fulfilled. If the composite FTC law (2) is designed according to (21) and (22), then there exists a positive constant $\varepsilon^{*}$ such that the origin of system (15) is asymptotically stable for any $\varepsilon \in\left(0, \varepsilon^{*}\right)$.

Proof: The closed-loop system (15) can be represented as

$$
\left\{\begin{array}{l}
\dot{x}=\bar{A}_{11} x+\bar{A}_{12} z+B_{1}\left(u_{a}+F_{a}\right)+D_{1} w \\
\varepsilon \dot{z}=\bar{A}_{21} x+\bar{A}_{22} z+B_{2}\left(u_{a}+F_{a}\right)+D_{2} w .
\end{array}\right.
$$

According to the variable transformation (23), system (24) can be rewritten in the following equivalent form:

$$
\left[\begin{array}{c}
\dot{\phi} \\
\varepsilon \dot{\psi}
\end{array}\right]=\left(\Theta_{0}+\varepsilon \Theta_{1}+\varepsilon^{2} \Theta_{2}\right)\left[\begin{array}{l}
\phi \\
\psi
\end{array}\right]+\left[\begin{array}{l}
\bar{B}_{1}\left(u_{a}+F_{a}\right)+\bar{D}_{1} w \\
\bar{B}_{2}\left(u_{a}+F_{a}\right)+\bar{D}_{2} w
\end{array}\right]
$$

where

$$
\Theta_{0}=\left[\begin{array}{cc}
\bar{A}_{0} & 0 \\
0 & \bar{A}_{22}
\end{array}\right], \Theta_{2}=\left[\begin{array}{cc}
0 & -\bar{H} \bar{L} \bar{A}_{0} \bar{H} \\
0 & \bar{L} \bar{A}_{0} \bar{H}
\end{array}\right], \Theta_{1}=\left[\begin{array}{cc}
-\bar{H} \bar{L} \bar{A}_{0} & \bar{A}_{0} \bar{H}-\bar{H} \bar{L} \bar{A}_{12} \\
\bar{L} \bar{A}_{0} & \bar{L}_{12} \bar{A}_{12}
\end{array}\right] .
$$

Choose the following Lyapunov function candidate $V(t)=\phi^{T} P_{0} \phi+\varepsilon \psi^{T} P_{2} \psi$. Then, the time derivative of $V(t)$ along the trajectories of system (24) in the coordinates $(\phi, \psi)$ is given by

$$
\begin{aligned}
\dot{V}(t)= & 2 \phi^{T} P_{0} \dot{\phi}+2 \psi^{T} P_{2} \varepsilon \dot{\psi} \\
= & {\left[\begin{array}{c}
\phi \\
\psi
\end{array}\right]^{T}\left(\Xi_{0}+\varepsilon \Xi_{1}+\varepsilon^{2} \Xi_{2}\right)\left[\begin{array}{l}
\phi \\
\psi
\end{array}\right]+2\left(\phi^{T} P_{0} \bar{B}_{1}+\psi^{T} P_{2} \bar{B}_{2}\right) u_{a} } \\
& +2\left(\phi^{T} P_{0} \bar{B}_{1}+\psi^{T} P_{2} \bar{B}_{2}\right) F_{a}+2\left(\phi^{T} P_{0} \bar{D}_{1}+\psi^{T} P_{2} \bar{D}_{2}\right) w,
\end{aligned}
$$


where

$$
\begin{aligned}
& \Xi_{0}=\left[\begin{array}{cc}
\operatorname{He}\left\{P_{0} \bar{A}_{0}\right\} & 0 \\
0 & \operatorname{He}\left\{P_{2} \bar{A}_{22}\right\}
\end{array}\right], \Xi_{2}=\left[\begin{array}{cc}
0 & -P_{0} \bar{H} \bar{L} \bar{A}_{0} \bar{H} \\
* & \operatorname{He}\left\{P_{2} \bar{L} \bar{A}_{0} \bar{H}\right\}
\end{array}\right], \\
& \Xi_{1}=\left[\begin{array}{cc}
-\operatorname{He}\left\{P_{0} \bar{H} \bar{L} \bar{A}_{0}\right\} & P_{0}\left(\bar{A}_{0} \bar{H}-\bar{H} \bar{L} \bar{A}_{12}\right)+\bar{L} \bar{A}_{0} P_{2} \\
* & \operatorname{He}\left\{P_{2} \bar{L} \bar{A}_{12}\right\}
\end{array}\right] .
\end{aligned}
$$

Substituting the compensate control law $u_{a}$ into the inequality above can deduce that

$$
\dot{V}(t) \leq\left[\begin{array}{c}
\phi \\
\psi
\end{array}\right]^{T}\left(\Xi_{0}+\varepsilon \Xi_{1}+\varepsilon^{2} \Xi_{2}\right)\left[\begin{array}{l}
\phi \\
\psi
\end{array}\right] \text {. }
$$

In fact, the following equation holds

$$
\begin{aligned}
\bar{A}_{0}= & \bar{A}_{11}-\bar{A}_{11} \bar{L}=\left(A_{11}+B_{1} K_{1}\right) \\
& -\left(A_{12}+B_{1} K_{2}\right)\left(A_{22}+B_{2} K_{2}\right)^{-1}\left(A_{21}+B_{2} K_{1}\right)=A_{0}+B_{0} K_{0} .
\end{aligned}
$$

Then, based on the condition (19), it can be obtained that $\operatorname{He}\left\{P_{0} \bar{A}_{0}\right\}<0$. Note also from the condition (20) that $\operatorname{He}\left\{P_{2} \bar{A}_{22}\right\}=\operatorname{He}\left\{P_{2}\left(A_{22}+B_{2} K_{2}\right)\right\}<0$. Therefore, it can be concluded that $\Xi_{0}<0$, which implies that there exists a sufficiently small $\varepsilon^{*}$ such that

$$
\Xi_{0}+\varepsilon \Xi_{1}+\varepsilon^{2} \Xi_{2}<0, \forall \varepsilon \in\left(0, \varepsilon^{*}\right)
$$

Therefore, the origin of system (15) is asymptotically stable for any $\varepsilon \in\left(0, \varepsilon^{*}\right)$. This ends the proof.

Remark 4: Theorem 2 gives a sufficient condition for stability of the closed-loop system, and the existence of SPP. The following result shows how to evaluate SPP.

Theorem 3: For a given positive constant $\bar{\varepsilon}>0$ and the selected gain matrices $K_{1}$ and $K_{2}$, if there exist $P_{0}>0$ and $P_{2}>0$ satisfying the following LMIs

$$
\begin{aligned}
\Xi_{0} & <0, \\
\Xi_{0}+\bar{\varepsilon} \Xi_{1} & <0, \\
\Xi_{0}+\bar{\varepsilon} \Xi_{1}+\bar{\varepsilon}^{2} \Xi_{2} & <0,
\end{aligned}
$$

then for any $\varepsilon \in(0, \bar{\varepsilon}]$, the system (15) is asymptotically stable under the proposed FTC law.

Proof: From the inequalities (30)-(32) and Lemma 1 in [31], the asymptotical stability of the closed-loop system (15) can be realized for any $\varepsilon \in(0, \bar{\varepsilon}]$, where $\bar{\varepsilon}$ is a predefined positive scalar. So the detailed proof is omitted here. This ends the proof.

According to Theorem 3, the upper bound of $\varepsilon$ can be obtained by solving the following optimization problem:

$$
\begin{aligned}
& \max _{P_{0}, P_{2}} \bar{\varepsilon} \\
& \text { s. t. }(30)-(32) .
\end{aligned}
$$

Similarly, when $\varepsilon$ is unknown and sufficiently small, the following $\varepsilon$-independent FTC law is presented for system (15):

$$
\bar{u}_{c}=u_{n c}-\frac{\hat{\Pi}^{T} \bar{F}}{\|\hat{\Pi}\|}-\frac{\hat{\Pi}^{T}\|\hat{\Psi}\| \bar{w}}{\|\hat{\Pi}\|^{2}},
$$

where $\hat{\Pi}=2\left(\hat{\phi}^{T} P_{0} \hat{B}_{1}+\hat{\psi}^{T} P_{2} \hat{B}_{2}\right), \quad \hat{\Psi}=2\left(\hat{\phi}^{T} P_{0} \hat{D}_{1}+\hat{\psi}^{T} P_{2} \hat{D}_{2}\right), \quad \hat{B}_{1}=B_{1}-\bar{H} B_{2}, \quad \hat{B}_{2}=B_{2}$, $\hat{D}_{1}=D_{1}-\bar{H} D_{2}, \hat{D}_{2}=D_{2}, \hat{\phi}=x$, and $\hat{\psi}=\bar{L} x+z$, the other notations are defined in (22).

Moreover, to avoid the chattering due to the discontinuous of the compensate control law, the revised composite FTC law $\hat{u}_{c}$ is developed as

$$
\hat{u}_{c}=\left\{\begin{array}{l}
\bar{u}_{c}, \text { if }\|\hat{\Pi}\| \geq \mu, \\
u_{n c}-\frac{\hat{\Pi}^{T} \bar{F}}{\mu}-\frac{\hat{\Pi}^{T}\|\hat{\Psi}\| \bar{w}}{\mu^{2}}, \text { otherwise. }
\end{array}\right.
$$

where $\mu$ is a predefined small positive constant. 


\section{NUMERICAL ILLUSTRATIONS}

In the section, two examples are proposed to show the effectiveness of the obtained results. In particular, Example 1 is related to the case of nonlinear SPS with unmatched disturbance, while Example 2 is related to that of the linear one with matched disturbance.

Example 1: Consider the nonlinear SPS (1) with the following parameters:

$$
\begin{aligned}
& f_{1}(x, z)=-x+(x+0.5) z, g_{1}(x, z)=0, \varrho_{1}(x, z)=\frac{|x| z}{1+z^{2}}, \\
& f_{2}(x, z)=-x^{2}+z, g_{2}(x, z)=1, \varrho_{2}(x, z)=\frac{x|z|}{1+x^{2}},
\end{aligned}
$$

the external disturbance $w=0.5 \sin (t)$ with the bound $\bar{w}=0.5, B_{x}=[-3,3], B_{z}=[-3,3]$, and the actuator bias fault $F_{a}=\cos (3 t)$. It is clear that Assumption 1 holds with $\bar{F}=1$.

\subsection{The Effectiveness of Theorem 1}

According to the proposed FTC scheme in Section 2, the reduced order subsystem and boundary layer subsystem of the nominal system in this example are given as follows, respectively,

$$
\dot{x}=-x+(x+0.5)\left(x^{2}-u_{s}\right),
$$

and

$$
\frac{d z}{d \tau}=-x^{2}+z+u_{s}+u_{f}
$$

For subsystem (36), design the slow control law $u_{s}=x^{2}$, and construct the Lyapunov function $V(x)=0.5 x^{2}$, it can be verified that the inequality (6) hold with $\alpha_{1}=2$. For subsystem (37), design the fast control law $u_{f}=-2 z$ and $W(x, z)=0.5 z^{2}$, it is easy to see that all conditions in Step 2 are satisfied with $\alpha_{2}=2$. Then, the composite control law for the nominal model is given as

$$
u_{n c}=u_{s}+u_{f}=x^{2}-2 z .
$$

Next, select the parameter $d=0.5, \beta_{1}=1.75, \beta_{2}=0$, and $\gamma=0$, it can be found that the interaction conditions (8) and (9) are satisfied. Meanwhile, design the compensate control law

$$
u_{a}=-\frac{z}{|z|}-\frac{0.5 z\left|\varrho_{2}(x, z) z\right|}{|z|^{2}} .
$$

With the proposed formula on upper bound of SPS, one obtains that $\varepsilon^{*}=+\infty$ since $d \alpha_{1} \alpha_{2}-$ $0.25(1-d) \beta_{1}^{2}>0$. Hence, it follows from Theorem 1 that for any $\varepsilon$, the system in Example 1 under the proposed composite control law is asymptotically stable. Fig. 1 shows the state trajectories and the control signal of the close-loop system in Example 1. Obviously, the proposed composite FTC law (38) and (39) can stabilize the system in this example. In the simulation, the initial state is chosen as $\left[\begin{array}{cc}x & z\end{array}\right]=\left[\begin{array}{ll}0 & 1\end{array}\right]$ and $\varepsilon=0.01$. However, it is easy to see that the compensate control law (39) lead to the chattering phenomenon.

\subsection{Discussion on The Chattering Phenomenon}

To eliminate the chattering while maintaining the stability of the controlled system, the following revised compensate control law is adopted to replace the control law (39).

$$
\hat{u}_{a}=\left\{\begin{array}{l}
-\frac{z}{|z|}-\frac{0.5 z\left|\varrho_{2}(x, z) z\right|}{|z|^{2}}, \text { if }|z| \geq \mu=0.05, \\
-\frac{z}{\mu}-\frac{0.5 z\left|\varrho_{2}(x, z) z\right|}{\mu^{2}}, \text { otherwise. }
\end{array}\right.
$$

Fig. 2 demonstrates the simulation results of the considered systems under the composite FTC law (38) and (40). It follows from Fig. 2 that the stability of the considered systems can be guaranteed and the chattering phenomenon mentioned above is eliminated.

\subsection{Comparison with Some Related Works}

The classical composite state feedback control law for nonlinear SPSs is considered in [24] and can be described as the control law (38) for this case. Fig. 3 shows that the state trajectories of the system 

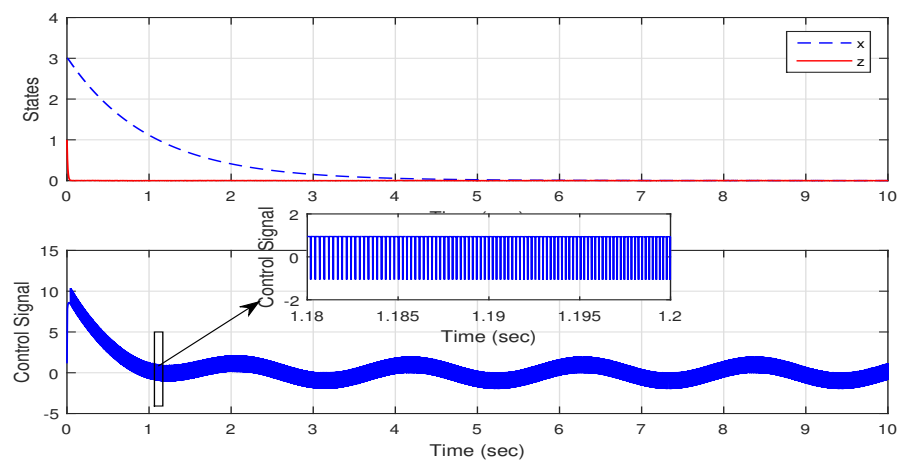

Figure 1. Simulation results of the closed-loop system in Example 1 under the control law (38) and (39).
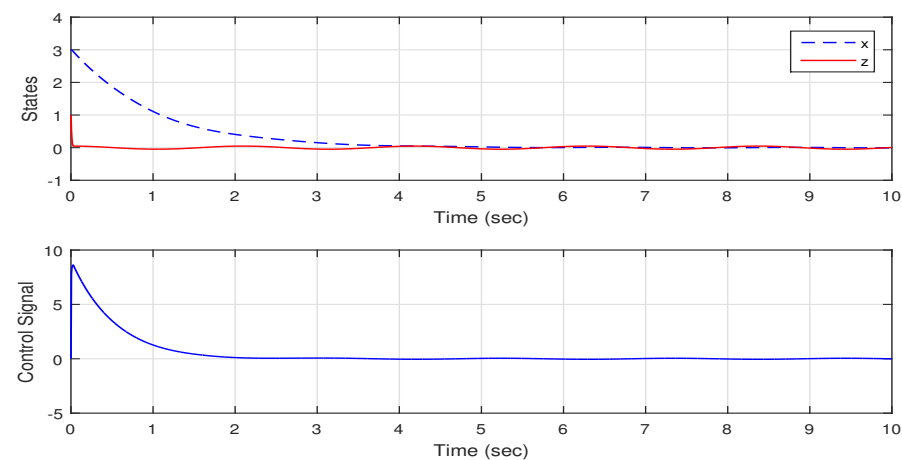

Figure 2. Simulation results of Example 1 under the control law (38) and (40).

under the control law (38) are divergent, which means that the classical composite state feedback control law is inapplicable to handle both the actuator fault and the disturbance.

Note that the FTC design for nonlinear SPSs under actuator faults is studied in [26] and the corresponding FTC law is provided. However, [26] does not take into account the external disturbances. The proposed FTC law in [26] cannot be applied here directly. Recently, [27] addressed the FTC design for linear SPSs face both actuator faults and external disturbances. But the results in [27] don't work for our case since the considered model is nonlinear one. In addition, both [26] and [27] do not provide the upper bound of SPP. Here, we can check that the SPP is smaller than the upper-bound proposed in Theorem 1 and consequently, the proposed FTC design can be applied.

Example 2: Let us consider the magnetic tape control system described in [32]. The dynamics can be represented as (15) where

$$
\begin{aligned}
& A_{11}=\left[\begin{array}{cc}
0 & 0.4 \\
0 & 0
\end{array}\right], A_{12}=\left[\begin{array}{cc}
0 & 0 \\
0.345 & 0
\end{array}\right], A_{21}=\left[\begin{array}{cc}
0 & -0.524 \\
0 & 0
\end{array}\right], \\
& A_{22}=\left[\begin{array}{cc}
-0.465 & 0.262 \\
0 & -1
\end{array}\right], B_{1}=D_{1}=\left[\begin{array}{l}
0 \\
0
\end{array}\right], B_{2}=D_{2}=\left[\begin{array}{l}
0 \\
1
\end{array}\right]
\end{aligned}
$$

the external disturbance $w(t)=\sin \left(\frac{\pi}{6} t\right)$ with the bound $\bar{w}=1$, and the actuator bias fault

$$
u_{F}=\left\{\begin{array}{l}
0, \text { if } 0 \leq t \leq 8 \\
5+2 \sin (0.1 t), t>8
\end{array}\right.
$$

One can easily check that Assumption 1 is satisfied with $\bar{F}=7$. 


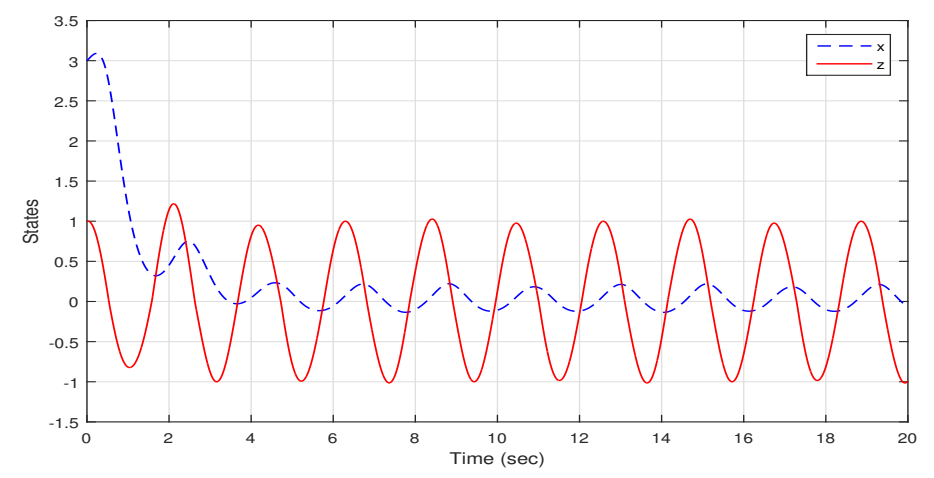

Figure 3. State response of the closed-loop system in Example 1 under the control law (38).

Based on the FTC scheme proposed in Section 3, it can be found that the slow and fast control laws are designed as $u_{s}=K_{0} x_{s}=-\left[\begin{array}{lll}21.3607 & 8.8482\end{array}\right] x_{s}$ and $u_{f}=K_{2} z_{f}=$ $-\left[\begin{array}{ll}0.7144 & 0.2241\end{array}\right] z_{f}$, respectively, then, the composite control law for the nominal model in this example is

$$
u_{n c}=-\left[\begin{array}{ll}
34.7469 & 15.1982
\end{array}\right] x-[0.7144 \quad 0.2241] z .
$$

Then, based on Theorem 3, choose the predefined value of singular perturbation parameter $\bar{\varepsilon}=0.1$, it can be found that all conditions all Theorem 3 are satisfied with

$$
P_{0}=\left[\begin{array}{cc}
21.6908 & 1.1513 \\
1.1513 & 2.2731
\end{array}\right], P_{2}=\left[\begin{array}{cc}
0.5152 & 0.4833 \\
0.4833 & 2.1324
\end{array}\right],
$$

which means that for any $\varepsilon \in(0,0.1]$, the asymptotical stability of the system in Example 2 can be ensured under the FTC law (34).

Moreover, by solving the obtained optimization problem (33), it can be found that $\bar{\varepsilon}=0.1666>$ 0.1 . By using Theorem 3 again, we know the controlled system in Example 2 is asymptotically stable for any $\varepsilon \in(0,0.1666]$. Fig. 4 depicts the simulation results of the Example 2 when the considered system imposed by the $\varepsilon$-independent FTC law (34), where the initial condition is chosen as $\left[\begin{array}{llll}-2 & 3 & -4 & 1\end{array}\right]^{T}$ and $\operatorname{SPP} \varepsilon=0.1$.

Note that the proposed FTC law will leads to the chattering phenomenon. To avoid such undesirable side-effects, by employing the proposed FTC law (35), the asymptotical stability of the system still can be maintained. Fig. 5 shows the state response and the control signal of the system in Example 2 under the continuous FTC law, where $\mu=0.05$.

Remark 5: Fig. 6 exhibits the state trajectories of the closed-loop system imposed by the composite law (42). It follows from Fig. 6 that the composite control law (42) cannot stabilize the system with the actuator fault. Note also that the FTC problem for a class of SPSs has been investigated in [28] and the sliding mode control design for SPSs in the presence of matched disturbances has been investigated in [32]. The aforementioned control laws depend on the SPP, therefore, they cannot be applied if the SPP is unknown even if sufficiently small.

\section{CONCLUSIONS}

In this paper, the FTC design problem for SPSs both actuator faults and external disturbances has been studied. First, a new composite FTC scheme has been proposed for nonlinear SPSs. Moreover, an $\varepsilon$-independent FTC scheme has been developed to ensure the desired stability of the controlled systems for unknown but sufficiently small SPP. The synthesis has been tailored to the case of linear SPSs by rewriting the results in term of LMIs. Moreover the computation of the SPP has 


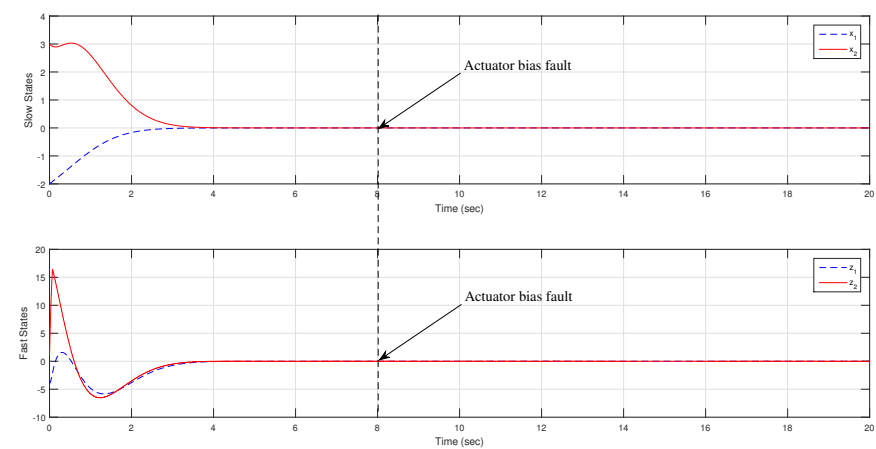

(a)

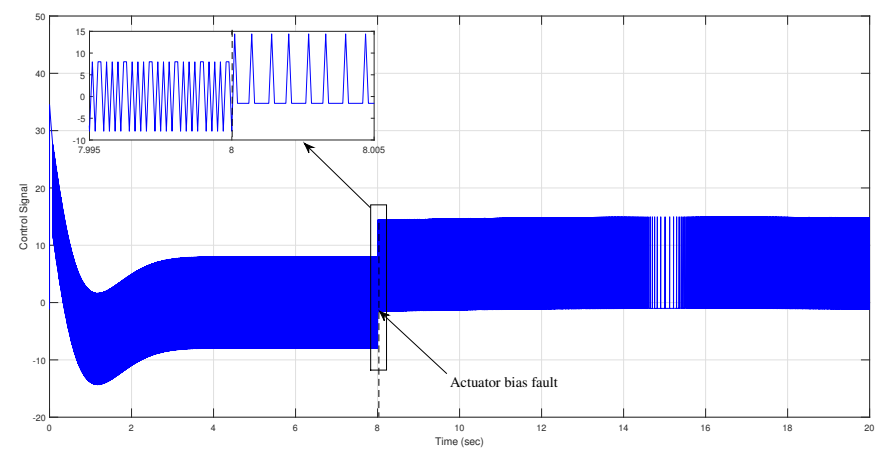

(b)

Figure 4. Simulation results of Example 2. (a) State response of the closed-loop system in Example 2 under the $\varepsilon$-independent FTC law; (b) Control signal.

been reformulated as a convex optimization problem. Two examples have been given to show the effectiveness of the proposed results. Future research will be focus on the FTC of singularly perturbed switched systems.

\section{ACKNOWLEDGEMENTS}

This work is supported by the National Natural Science Foundation of China under Grants 61903147 and 61773172, the Postdoctoral Science Foundation of China under Grant 2019M652644, the Natural Science Foundation of Hubei Province of China (2017CFA035), and the Fundamental Research Funds for the Central Universities (2018KFYYXJJ119).

\section{REFERENCES}

1. Xiao-Jian Li and Guang-Hong Yang. Robust adaptive fault-tolerant control for uncertain linear systems with actuator failures. IET Control Theory \& Applications, 6(10):1544-1551, 2012.

2. Li-Ying Hao, Ju H Park, and Dan Ye. Integral sliding mode fault-tolerant control for uncertain linear systems over networks with signals quantization. IEEE Transactions on Neural Networks and Learning Systems, 28(9):20882100, 2017.

3. Shuyi Xiao and Jiuxiang Dong. Robust adaptive fault-tolerant tracking control for uncertain linear systems with actuator failures based on the closed-loop reference model. IEEE Transactions on Systems, Man, and Cybernetics: Systems, 2018. DOI: 10.1109/TSMC.2018.2876125. 

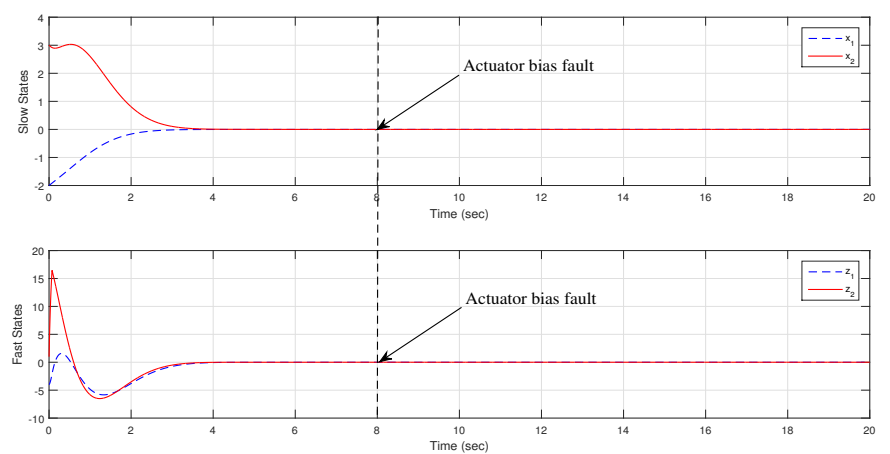

(a)

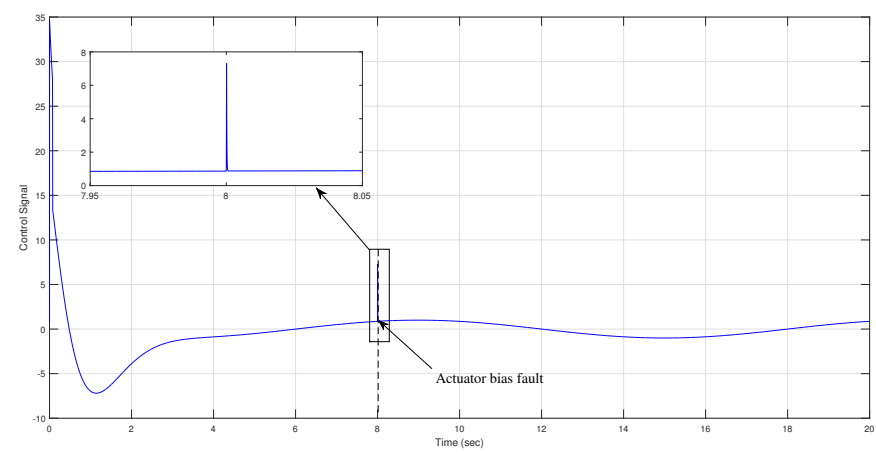

(b)

Figure 5. Simulation results of Example 2. (a) State response of the closed-loop system in Example 2 under the continuous FTC law; (b) Control signal.

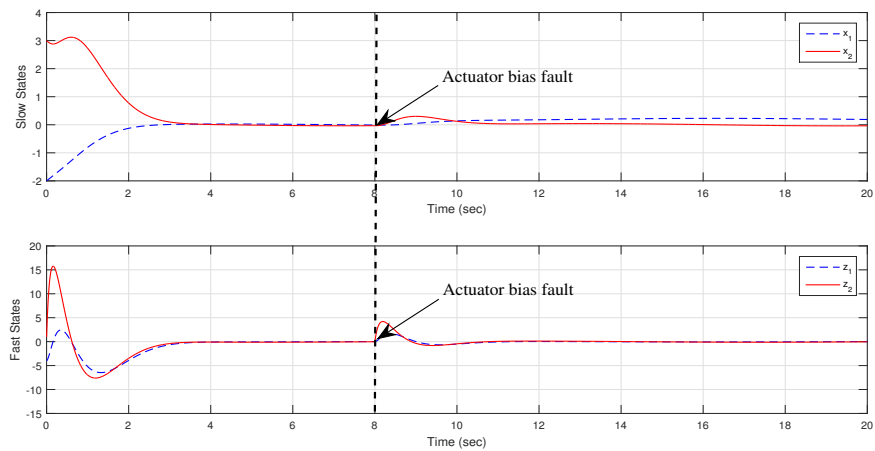

Figure 6. State response of the closed-loop system in Example 2 under the control law (42).

4. Bing Xiao, Xuebo Yang, Hamid Reza Karimi, and Jianbin Qiu. Asymptotic tracking control for a more representative class of uncertain nonlinear systems with mismatched uncertainties. IEEE Transactions on Industrial Electronics, 66(12):9417-9427, 2019.

5. Lu Cao and Bing Xiao. Exponential and resilient control for attitude tracking maneuvering of spacecraft with actuator uncertainties. IEEE/ASME Transactions on Mechatronics, 24(6):2531-2540, 2019.

6. Li-Bing $\mathrm{Wu}, \mathrm{Ju} \mathrm{H}$ Park, and Nan-Nan Zhao. Robust adaptive fault-tolerant tracking control for nonaffine stochastic nonlinear systems with full-state constraints. IEEE Transactions on Cybernetics, 2019. DOI: 10.1109/TCYB.2019.2940296.

7. Lei Liu, Yan-Jun Liu, and Shaocheng Tong. Neural networks-based adaptive finite-time fault-tolerant control for a class of strict-feedback switched nonlinear systems. IEEE Transactions on Cybernetics, 49(7):2536-2545., 2018. 
8. Li Tang, Dan Ma, and Jun Zhao. Neural networks-based active fault-tolerant control for a class of switched nonlinear systems with its application to RCL circuit. IEEE Transactions on Systems, Man, and Cybernetics: Systems, 2018. DOI: 10.1109/TSMC.2018.2847283.

9. Hao Yang, Hong Li, Bin Jiang, and Vincent Cocquempot. Fault tolerant control of switched systems: a generalized separation principle. IEEE Transactions on Control Systems Technology, 27(2):553-565, 2018.

10. Li-Bing Wu and Ju H Park. Adaptive fault-tolerant control of uncertain switched nonaffine nonlinear systems with actuator faults and time delays. IEEE Transactions on Systems, Man, and Cybernetics: Systems, 2019. DOI: 10.1109/TSMC.2019.2894750.

11. Palanisamy Selvaraj, Boomipalagan Kaviarasan, Rathinasamy Sakthivel, and Hamid Reza Karimi. Fault-tolerant SMC for Takagi-Sugeno fuzzy systems with time-varying delay and actuator saturation. IET Control Theory \& Applications, 11(8):1112-1123, 2017.

12. Jing-Jing Yan, Guang-Hong Yang, and Xiao-Jian Li. Adaptive fault-tolerant compensation control for T-S fuzzy systems with mismatched parameter uncertainties. IEEE Transactions on Systems, Man, and Cybernetics: Systems, 2018. DOI: 10.1109/TSMC.2018.2854630.

13. Dan Ye, Na-Na Diao, and Xin-Gang Zhao. Fault-tolerant controller design for general polynomial-fuzzy-modelbased systems. IEEE Transactions on Fuzzy Systems, 26(2):1046-1051, 2018.

14. Ming Liu, Peng Shi, Lixian Zhang, and Xudong Zhao. Fault-tolerant control for nonlinear markovian jump systems via proportional and derivative sliding mode observer technique. IEEE Transactions on Circuits and Systems I: Regular Papers, 58(11):2755-2764, 2011.

15. Xiaohang Li, Choon Ki Ahn, Dunke Lu, and Shenghui Guo. Robust simultaneous fault estimation and nonfragile output feedback fault-tolerant control for Markovian jump systems. IEEE Transactions on Systems, Man, and Cybernetics: Systems, 49(9):1769-1776, 2019.

16. Dong Zhao, Hamid Reza Karimi, Rathinasamy Sakthivel, and Yueyang Li. Non-fragile fault-tolerant control for nonlinear Markovian jump systems with intermittent actuator fault. Nonlinear Analysis: Hybrid Systems, 32:337$350,2019$.

17. Zhiqiang Zuo, Daniel WC Ho, and Yijing Wang. Fault tolerant control for singular systems with actuator saturation and nonlinear perturbation. Automatica, 46(3):569-576, 2010.

18. Zhiqiang Zuo, Yijing Wang, and Wenlong Yang. $L_{2}$-gain fault tolerant control of singular Lipschitz systems in the presence of actuator saturation. International Journal of Robust and Nonlinear Control, 25(12):1751-1766, 2015.

19. Xiaozheng Jin, Shaofan Wang, Jiahu Qin, Wei Xing Zheng, and Yu Kang. Adaptive fault-tolerant consensus for a class of uncertain nonlinear second-order multi-agent systems with circuit implementation. IEEE Transactions on Circuits and Systems I: Regular Papers, 65(7):2243-2255, 2017.

20. Mohsen Khalili, Xiaodong Zhang, Marios M Polycarpou, Thomas Parisini, and Yongcan Cao. Distributed adaptive fault-tolerant control of uncertain multi-agent systems. Automatica, 87:142-151, 2018.

21. Chengshan Wang, Xiaopeng $\mathrm{Fu}$, Peng Li, Jianzhong Wu, and Liwei Wang. Multiscale simulation of power system transients based on the matrix exponential function. IEEE Transactions on Power Systems, 32(3):1913-1926, 2017.

22. Fathi Ghorbel and Mark W Spong. Integral manifolds of singularly perturbed systems with application to rigid-link flexible-joint multibody systems. International Journal of Non-Linear Mechanics, 35(1):133-155, 2000.

23. Chang Hyeong Lee and Hans G Othmer. A multi-time-scale analysis of chemical reaction networks: I. deterministic systems. Journal of Mathematical Biology, 60(3):387, 2010.

24. Petar Kokotovic, Hassan K Khalil, and John O'reilly. Singular Perturbation Methods in Control: Analysis and Design. SIAM, Philadelphia, PA, 1999.

25. Nouceyba Abdelkrim, Adel Tellili, and Mohamed Naceur Abdelkrim. Additive fault tolerant control applied to delayed singularly perturbed system. Journal of Software Engineering and Applications, 5(04):217, 2012.

26. Adel Tellili, Aymen Elghoul, and Mohamed Naceur Abdelkrim. Additive fault tolerant control of nonlinear singularly perturbed systems against actuator fault. Journal of Electrical Engineering, 68(1):68-73, 2017.

27. Adel Tellili, Nouceyba Abdelkrim, Amina Challouf, and Mohamed-Naceur Abdelkrim. Adaptive fault tolerant control of multi-time-scale singularly perturbed systems. International Journal of Automation and Computing, 15(6):736-746, 2018.

28. Jing Wang, Kun Liang, Xia Huang, Zhen Wang, and Hao Shen. Dissipative fault-tolerant control for nonlinear singular perturbed systems with Markov jumping parameters based on slow state feedback. Applied Mathematics and Computation, 328:247-262, 2018.

29. Hao Yang, Bin Jiang, and Vincent Cocquempot. Fault tolerant control design for hybrid systems. Springer Science \& Business Media, 2010.

30. Hassan K Khalil. Nonlinear systems. Englewood Cliffs, NJ, USA: Prentice Hall, 2002.

31. Yan-Wu Wang, Wu Yang, Xiao-Kang Liu, and Wu-Hua Chen. Dissipativity of singularly perturbed Lur'e systems. IEEE Transactions on Circuits and Systems II: Express Briefs, 66(9):1532-1536, 2019.

32. Thang Nguyen, Wu-Chung Su, and Zoran Gajic. Variable structure control for singularly perturbed linear continuous systems with matched disturbances. IEEE Transactions on Automatic Control, 57(3):777-783, 2012. 